\title{
LA CONFIANZA INTERPERSONAL ENTRE PROFESORES Y DIRECTORES DE ESCUELAS: UN PILAR FUNDAMENTAL PARA LA IMPLEMENTACIÓN DE POLÍTICAS Y PROGRAMAS EDUCATIVOS ${ }^{\mathrm{I}}$
}

\begin{abstract}
Alicia Razeto P.*
RESUMEN:

Este artículo aborda la importancia de las relaciones de confianza -especialmente entre profesores y director- al interior de las escuelas como recurso para la implementación de las políticas educacionales creadas por los gobiernos, y que presionan el quehacer cotidiano de las escuelas. Las relaciones de confianza se constituyen como capacidades de implementación, que pueden contribuir a aumentar la efectividad de los colegios en la puesta en marcha de los programas educativos.
\end{abstract}

Palabras clave: relaciones sociales, confianza, implementación de políticas educacionales, escuelas efectivas.

\section{INTERPERSONAL TRUST BETWEEN TEACHERS AND SCHOOL PRINCIPALS: A CORNERSTONE FOR THE IMPLEMENTATION OF EDUCATIONAL POLICIES AND PROGRAMS}

ABSTRACT:

This paper analyze the importance of the trust relationships inside de Chileans school's -specially between teachers, and between teachers and principal- as a resource for the implementation of educational policies that press the habitual work of school's. The trust relationships are capabilities of implementation, which can to promote the efficiency of school's in the realization of educational programs.

Keywords: social relationships, trust, implementation of educational policies, effective's schools.

I Artículo asociado al Proyecto FONDECYT n ${ }^{\circ} 1095186$ año 2009.

* Alicia Razeto es trabajadora social, Magíster en Gestión y Políticas Públicas de la Universidad de Chile y Candidata a Doctor en Educación de la Pontificia Universidad Católica de Chile. Actualmente se desempeña como profesora de la Escuela de Trabajo Social de la Pontificia Universidad Católica de Chile. Su correo electrónico es arazeto@uc.cl 


\section{La implementación de políticas educacionales en las escuelas}

Durante los últimos años, las escuelas chilenas han estado en el centro de las preocupaciones del Estado y de la sociedad civil, por el llamado a mejorar la calidad educativa en el marco de un país que aspira al desarrollo humano y a su integración en la OCDE. Diversas políticas y programas educativos han sido elaborados y puestos en marcha al interior de escuelas como estrategia para avanzar hacia la calidad y equidad del sistema. Sin embargo, se ha constatado en la implementación de dichos programas que un factor de suma importancia en el éxito de este proceso es que la escuelas tengan capacidades para la ejecución de los programas, junto a que los cambios educativos que ellos promueven tengan sentido para los actores de la comunidad escolar.

En el entendido de lo importante que es observar hacia el interior de las escuelas cuando se implementan políticas educativas, este artículo pone la mirada en lo que sucede al interior de las escuelas, en un escenario marcado por las fuertes presiones y exigencias que representan las políticas educativas en torno al mejoramiento de la calidad de la educación para los actores de la comunidad escolar. En este artículo, se plantea que las relaciones sociales entre los actores de la comunidad escolar constituyen un pilar fundamental para la implementación de las políticas educativas, ya que "el vínculo cotidiano entre las personas es la trama básica que hace a la sociedad" (PNUD, 1998:I40), y especialmente, por que el capital social del que la escuela dispone (relaciones fluidas, buen clima organizacional y compromiso de los profesores) es un factor que ayuda a conseguir buenos resultados educacionales (Raczynski y Muñoz, 2005). De este modo, planteamos que el desarrollo de relaciones sociales de confianza al interior de las escuelas se convierte en un fuerte pilar sobre el cual se ponen en marcha las políticas y programas educativos.

La implementación de políticas educacionales en las escuelas ejerce presión y fuertes exigencias a sus miembros, afectando las labores de quienes se desempeñan en ella, demandándoles un mejor desempeño 
al director, a los profesores y también a los estudiantes, cada uno en el rol que le corresponde. La labor de todos los miembros de la organización escolar, particularmente la del director y profesores, se encuentra ligada y es altamente dependiente y vulnerable a la del otro (Bryk y Schneider, 2002). Por ello, más que en cualquier otro tipo de organización, las relaciones de confianza que se desarrollan en las escuelas se convierten en pilares fundamentales que sustentan la posibilidad de procesamiento de estas presiones.

En este planteamiento, nos apoyamos en Putnam (2000), quien señala que la reciprocidad, honestidad y la confianza contribuyen a lubricar las inevitables fricciones de la vida social. De este modo, podemos concebir que las relaciones sociales que descansan en ese tipo de valores, ayudan a procesar las presiones y a implementar acciones colectivas para la puesta en marcha de los planes y programas educacionales provenientes desde el gobierno. En otras palabras, podemos plantear que el capital social de la escuela, posee una capacidad fundamental para mejorar la implementación de las políticas y por tanto, incidir en la efectividad del funcionamiento de una organización escolar. En esto, nos basaremos en estudios internacionales que asocian confianza con efectividad escolar (Uline, Millar y Tschannen-Moran, I998; Tschannen-Moran y Hoy, 2003), entendiendo que la confianza es la base desde la cual la efectividad de la escuela es construida (Cunningham y Gresso, I993).

En este artículo, realzamos la particular importancia de una de las dimensiones del capital social y de las relaciones sociales, catalogada como "un hecho básico de la vida social” (Luhmann, 1996:5), como "un elemento básico en la configuración de las relaciones interpersonales” (PNUD, I998:I4I): la confianza.

Planteamos que la presencia de relaciones de confianza al interior de la escuela entre director y profesores, y entre profesores, contribuye al buen procesamiento de las políticas educativas. En consecuencia, contribuye a su buena implementación, al facilitar las relaciones de cooperación y el trabajo colectivo con miras a alcanzar los objetivos y propósitos de la política. A la vez, la presencia de confianza en 
las relaciones favorece el desarrollo de la identidad en los miembros de la escuela, ya que "un alto grado de confianza interpersonal es expresión del vigor del "nosotros" que regula nuestras relaciones. Ello permite descansar en la regulación social del vínculo y profundizar en la particularidad de la relación inmediata” (PNUD, I998:I4I). Por todo esto, y basados en la premisa de que la confianza es un mecanismo de reducción de la complejidad social (Luhmann, I996), podemos sugerir que el desarrollo de relaciones de confianza promueve la implementación de políticas educativas, lo que contribuye al buen funcionamiento de la organización escolar y a su efectividad.

\section{Sobre el concepto de confianza}

La confianza ha sido más trabajada por la teoría sociológica que por la educativa, aunque no con la profundidad esperada si se considera la centralidad que actualmente se le asigna en la comprensión de las relaciones sociales. Si bien "ha sido incorporada en varios modelos de relaciones sociales, la confianza rara vez ha sido explícitamente cuestionada o estudiada. Las ciencias sociales modernas no han contribuido significativamente a nuestra comprensión del concepto de confianza y de las condiciones bajo las cuales las relaciones de confianza se desarrollan o tienen problemas para sobrevivir" (Misztal, 1996:2). Este exiguo aporte de las ciencias sociales en el estudio de la confianza se contradice con la importancia que los estudiosos del tema le asignan como componente indispensable para el desarrollo del orden y estabilidad social (Luhmann, 1996; Seligman, I997; Misztal, 1996; Sztompka, 1999). Asimismo, la escasa contribución de la teoría educativa al estudio de las relaciones de confianza se contrapone con la influencia que ellas pueden tener en el aprendizaje de los estudiantes y la efectividad de la escuela en la implementación de políticas educativas.

Para Sztompka (1999), recién en la década de los noventa el problema de la confianza ha empezado a suscitar atención sociológica. Desde una preocupación marginal de algunos estudiosos a comienzo de los ochenta (Luhmann, I979; Barber, I983) se ha girado hacia 
una empresa intelectualmente rica con un número no despreciable de contribuciones. Durante los noventa, el concepto de confianza se ha presentado con una creciente frecuencia y prominencia en la teorización acerca de la sociedad moderna (Misztal, 1996:2). Por ejemplo, autores como Giddens (1990) y Beck (1992) abordan la confianza en relación a la modernización de las sociedades, la globalización y el riesgo, mientras que Luhmann (1996) analiza la confianza como mecanismo de reducción de la complejidad social.

En el escenario contemporáneo, la preocupación sobre la idea de la sociedad civil, representa un claro llamado a definir nuevos términos de confianza generalizada en las sociedades democráticas modernas (Seligman, 1997). Asimismo, el cuestionamiento sobre los caminos para avanzar en el desarrollo ha encontrado en la teoría sobre el capital social respuestas que concuerdan en atribuir mayores grados de protagonismo a las relaciones sociales, y particularmente a la confianza, como valores cruciales en el desarrollo de las naciones. En el campo educativo, existen estudios que aplican la teoría del capital social a la escuela, encontrando en él un factor coadyuvante en la comprensión del éxito o fracaso de las escuelas (Coleman, 2003; Schuller, Baron y Field, 2000).

Comenzaremos revisando el concepto de confianza de uno de los sociólogos contemporáneos más connotados del último tiempo: Niklas Luhmann (1927-1998). La confianza es concebida por Luhmann (1979:4) "en el más amplio sentido de la confianza en las expectativas de uno, como un hecho básico de la vida social”, que le permite a los individuos movilizarse en el mundo, o bien paralizarlos ante su ausencia: "una completa ausencia de confianza le impediría (al hombre) incluso levantarse en la mañana. Sería víctima de un sentido vago de miedo y de temores paralizantes. Incluso no sería capaz de formular una desconfianza definitiva y hacer de ello un fundamento para medidas preventivas, ya que esto presupondría confianza en otras direcciones. Cualquier cosa y todo sería posible”(Luhmann, 1996:5). Esta noción sobre la confianza aplicada a la escuela revela lo central que es su presencia, y al mismo tiempo, lo difícil que es para una 
organización educativa funcionar cotidianamente sin la presencia de un patrón mínimo de confianza en las relaciones entre los miembros que la componen. Considerando que la confianza es necesaria para las relaciones de cooperación y trabajo colectivo, es posible afirmar que sin la presencia de un mínimo de confianza imperaría el caos al interior de las escuelas.

Luhmann plantea que "donde hay confianza hay aumento de posibilidades para la experiencia y la acción, hay un aumento de la complejidad del sistema social y también del número de posibilidades que pueden reconciliarse con su estructura, porque la confianza constituye una forma más efectiva de reducción de la complejidad”. Al existir confianza en las relaciones entre los miembros de la organización escolar, se aumenta las posibilidades de acción conjunta y de experiencia, contribuyendo a generar nuevas y mejores estrategias de mejoramiento de los aprendizajes, y nuevas y mejores estrategias para implementar las políticas educativas. Esto aumenta la complejidad de la organización, pero al mismo tiempo la disminuye, puesto que la complejidad puede encontrar respuesta en una estructura escolar clara y conocida, con relaciones sociales basadas en la certeza y la confianza en la acción del otro.

El autor realza la importancia de la confianza interpersonal, al indicar que "basándose en la familiaridad con el mundo cotidiano, la confianza es principalmente una confianza interpersonal. Sirve para superar el elemento de incertidumbre en el comportamiento de otras personas, que se experimenta como la imposibilidad de predecir el cambio de un objeto" (Luhmann, I996:36). También destaca a la confianza en el sistema como una forma de confianza más amplia que la interpersonal, que se origina por el aumento de la complejidad en donde la otra persona entra en juego como coautor de esa complejidad y su reducción. La confianza en el sistema "implica renunciar, como un riesgo consciente, a alguna posibilidad de mayor información, así como también a una indiferencia cuidadosa y al continuo control de los resultados. La confianza en el sistema no es sólo aplicable a los sistemas sociales, sino también a otras personas como sistemas personales" (Luhmann, 1996:37). 
En el mundo de las escuelas, la confianza interpersonal entre profesores, y entre profesores y el director de la escuela, se convierte en un atributo de gran valor en las relaciones, al permitir superar la incertidumbre que el profesor tiene con el comportamiento de sus colegas y del director, y que el director tiene con respecto al comportamiento de sus profesores.

Luhmann (1996:66) concibe a la confianza interpersonal como "la expectativa generalizada de que otro manejará su libertad, su potencial perturbador para la acción diversa, manteniendo su personalidad o más bien manteniendo la personalidad que ha mostrado y hecho socialmente visible. El que se mantiene con lo que ha permitido que se sepa acerca de él, ya sea consciente o inconscientemente, es acreedor de la confianza". Es importante destacar que "las posibilidades de acción aumentan proporcionalmente al aumento de la confianza -la confianza en nuestra autopresentación y en la interpretación de otras personas de ésta-. Cuando tal confianza se ha establecido, nuevas formas de comportamiento son posibles: chistes, iniciativas informales, brusquedad, interrupciones verbales, silencios oportunos, la elección de temas delicados, etc. Cuando la confianza es examinada y probada en esta forma, puede acumularse a manera de capital de confianza” (Luhmann, 1996:68).

La acumulación de capital de confianza en una escuela puede convertirse en un recurso facilitador de las relaciones de trabajo cotidianas, ayudando a disipar dudas respecto a comportamientos no esperados a los estándares existentes en una organización, y propiciando la proliferación de más alternativas de acción respecto a problemáticas o situaciones presentes en la escuela y que atañen al equipo de profesores, tales como la implementación de un nuevo programa educativo, la definición de estrategias de mejoramiento de aprendizaje, la instauración de nuevos mecanismos de evaluación de aprendizaje, entre otros.

La confianza es necesaria para que la sociedad, sus sistemas y en particular la escuela funcionen. No es por casualidad el hecho que la confianza aparezca como un rasgo de las relaciones en el mundo 
social. Sztompka (1999) resalta que la importancia de la confianza se observa no en el mundo natural, sino que en el social: "tenemos que coexistir con otros, coordinar nuestras acciones con ellos, y en más avanzadas etapas de la sociedad humana, cooperar con ellos (Sztompka, 1999:22)”. El problema que subyace a esto, según el autor, es que el ambiente social posee un alto grado de incerteza y descontrol, y a la vez, las personas se generan expectativas frente a la acción de los otros. A menudo estas expectativas no son correspondidas por los actos de las personas involucradas, lo que puede destruir las confianzas y por tanto, reducir las posibilidades de acción conjunta.

El concepto de confianza puede ser comprendido desde distintos puntos de vista. Misztal (1996) plantea que desde la literatura sociológica, se conceptualiza a la confianza como una propiedad de los individuos, de las relaciones sociales, y del sistema social para explicar el comportamiento basado en acciones y orientaciones a nivel individual. El primer enfoque "pone énfasis en los sentimientos, emociones y valores individuales. La confianza es vista como una función de las variables de personalidad de los individuos" (Deutsch 1958; Cole 1973; Wolfe 1976 citado en Misztal, 1996:I4). La autora manifiesta que en esta perspectiva, la confianza suele confundirse o relacionarse con la mentalidad cooperativa, la honestidad, la lealtad, sinceridad, esperanza o altruismo.

En el segundo enfoque, la confianza es vista como un atributo colectivo, aplicable a la fábrica institucional de la sociedad. Por ejemplo, el modo de producción fordista es descrito como un sistema de baja confianza (Fox 1974 citado en Misztal), mientras que el modelo post-fordista de la organización de la manufactura es denominado como un sistema de confianza dependiente (Sabel I989; Hirst and Zetlin I99I citado en Misztal, I996:I4). La autora señala que en este enfoque la confianza puede ser vista como un recurso social que puede ser usado para lograr metas organizacionales.

En el tercer enfoque, la confianza es vista como un bien público valorado, sostenido por las acciones de los miembros de una sociedad 
(Misztal, 1996). En este enfoque se pueden destacar las descripciones hechas por Tocqueville sobre la comunidad cívica, y los estudios de Putnam sobre el rol de la confianza y el capital social en el desarrollo democrático en el norte de Italia. Según Misztal (1996) en esta perspectiva es también posible reconocer los trabajos de Luhmann (1979) y Barber (1983) que ven la confianza como una dimensión de la estructura social que no puede ser estudiada sólo a nivel psicológico o institucional, ya que la confianza los permea a ambos.

En el análisis de las relaciones de confianza al interior de las escuelas, es posible usar los tres enfoques sin excluir a ninguno de ellos, en tanto la confianza es un atributo de las personas que les permite a profesores y director discernir acerca del comportamiento y reputación del otro. Es un atributo de las relaciones sociales en tanto más confianza permite lubricar la interacción cotidiana entre profesores y director, conduciendo al cumplimiento de los propósitos de la escuela. Y finalmente, es un atributo de la sociedad, en tanto una sociedad con más confianza se desarrolla más rápida y equitativamente entre sus miembros que una que no lo es.

De cualquier modo, una de las cuestiones que caracteriza a la confianza es que opera en situaciones orientadas a un futuro no manipulable, frente al cual existe incertidumbre acerca de los resultados de la acción de los involucrados. Sztompka (I999) desarrolla la idea de confianza en el contexto de la acción humana, resaltando que su más relevante rasgo es la orientación hacia un futuro que es incierto, produciéndose una diferencia entre lo que se hace y los resultados deseados o indeseados de las acciones emprendidas. Esto lleva a que los individuos intenten predecir y controlar el futuro, con mayores o menores grados de certeza en la predicción, "cuando estamos prácticamente ciertos acerca del futuro, es obvio que no necesitamos la confianza (...) pero en muchas otras ocasiones nosotros podemos predecir solo con algún grado de probabilidad (Sztompka, 1999:19)" en estos casos la confianza es requerida. En el contexto escolar, cargado de situaciones imprevistas e inciertas, con escuelas fuertemente orientadas al futuro en el sentido de avanzar 
hacia escenarios de mayor calidad y equidad de los aprendizajes, se hace visible la importancia de que los profesores y director actúen con confianza hacia la labor del otro.

Según Misztal (1996) es posible encontrar diversas definiciones de confianza contenidas en los propios desarrollos teóricos de sus autores, así como también es posible rastrear el concepto en enciclopedias y diccionarios, cada una con sus propias connotaciones. La autora plantea que la connotación más antigua sobre la confianza es aquella relacionada a la fe o "la confianza en un poder supranatural en el cual el hombre se siente como dependiente (Morgan I9I2 citado en Misztal, I996:I5)”. Este tipo de confianza está presente en todas las creencias religiosas en tanto la confianza en Dios sería una fuente de salvación. Para Giddens (I990, citado en Misztal I996:I5) la confianza es una forma de fe, en la cual "la confianza vertida en una resultado probable expresa un compromiso con algo más que solo un entendimiento cognitivo”.

Desde la perspectiva económica (Misztal, I996), la confianza aparece como una característica del mercado, puesta en la moneda como medio de intercambio, y en la confianza en la habilidad e intención de un comprador a pagar en el futuro por bienes obtenidos en el presente sin pago presente.

Para los análisis antropológicos, "los cuales tienden a enfatizar los estándares normativos que sustentan el intercambio, muestran la importancia de la reciprocidad como obligaciones convincentes y prolongamiento de asociaciones (Shalins 1972 citado en Misztal, 1996:I6)”. La confianza juega un rol significativo en cualquier intercambio donde cada parte tiene una expectativa clara del otro, y donde hay un lapso de tiempo entre el intercambio de bienes o servicios (Misztal, 1996). Desde la antropología, se ha estudiado que el intercambio de regalos está basado en la confianza, sin embargo, menos sencilla ha resultado la relación entre los intercambios cooperativos y la confianza: "la cooperación es vista como un producto de la confianza más que como una fuente de ella, por lo 
tanto, la falta de confianza puede ser el resultado de otros factores y no sólo de la ausencia de confianza (Misztal, 1998:17)”.

Esto nos lleva a la visión de la confianza como mecanismo social, y a la atención de las creencias, motivaciones, y sus fuentes, presentes en ella. "Las aproximaciones sociológicas, generalmente argumentan que las relaciones e interacciones sociales son el punto de origen de las motivaciones y creencias" (Misztal, 1996:19). Las relaciones sociales y las obligaciones inherentes a ellas serían los factores responsables de la producción de la confianza. Aquí, la materia moral aparece como relevante, en tanto "tenemos reputaciones que proteger, tareas cooperativas que realizar, legados que dejar, otros que amar y carreras que seguir" (Wolfe 1989 citado en Misztal, 1996:19). De esta manera, según la autora, reconciliando nuestras necesidades con las de otros, organizando y reflexionando en nuestras relaciones con otros, construimos nuestras expectativas y creencias acerca de los otros, y ello influencia de manera importante nuestras actitudes de confianza.

El hecho de que los profesores y el director de una escuela compartan la misión de la educación de los estudiantes se convierte en una premisa básica para el desarrollo de la confianza en las relaciones sociales entre sus miembros. Se trataría del componente moral de la confianza (Uslaner, 2002) en el entendido que "la confianza en otras personas está basada en un postulado ético fundamental: que las otras personas comparten tus valores fundamentales. Ellos no necesariamente están de acuerdo política o religiosamente contigo. Pero en algún nivel fundamental, las personas aceptan el argumento de que ellos tienen en común vínculos que desarrollan cooperación vital. Y esos vínculos comunes descansan en suposiciones acerca de la naturaleza humana" (Uslaner, 2002:2). Según el mismo autor, estas suposiciones consisten en creer que el mundo es un lugar benefactor compuesto por personas que son bien intencionadas y por lo tanto confiables, "tan bueno como el mundo es, las cosas serán hechas mejor y nosotros podemos hacer esto entonces; tenemos obligaciones hacia el otro" (Uslaner, 2002:2). 


\section{Los beneficios de las relaciones de confianza al interior de la escuela}

Kramer (1999) identifica tres beneficios de la confianza para las organizaciones -a nivel tanto individual como colectivo- basándose en Putnam (1993) y Fukuyama (1995). Estos son: i) reduce los costos de transacción, ya que la confianza es un mecanismo efectivo y de bajo costo para resolver situaciones en las que existen dilemas en la elección, ii) aumenta la sociabilidad espontánea entre los miembros de una organización, referida a las múltiples formas de comportamiento cooperativo y altruista para fomentar el bien de la organización y las metas colectivas, iii) facilita formas apropiadas de deferencia hacia las autoridades organizacionales, lo que contribuye al eficiente desempeño organizacional.

Estos beneficios de la confianza para las organizaciones pueden ser aplicados a las escuelas, ya que la confianza puede reducir los costos de transacción en situaciones en las que exista algún tipo de dificultad, sin ser necesario acudir a la elaboración de contratos o compromisos legales, siendo suficiente depositar confianza en las personas involucradas. Por otra parte, la confianza al interior de una escuela ayuda a generar espacios de sociabilidad que pueden ser usados para promover la cooperación entre las personas, cuestión de suma relevancia para el eficaz funcionamiento de una escuela. Aunque hay que tener presente que "la confianza puede ser vista como un mecanismo para resolver el problema de la cooperación sólo cuando las personas cooperan independientemente de las sanciones y recompensas” (Misztal, 1996:99). De esta forma, la promoción de la confianza puede ayudar a crear las condiciones para aumentar los vínculos de solidaridad, tolerancia y legitimidad (Misztal, 1996). A su vez, la confianza puede contribuir a fortalecer el liderazgo del director de una escuela, conduciendo a formas de relación basadas en el respeto y valoración de la labor del otro, lo que motiva el buen desempeño de los miembros de una organización.

Una de las investigaciones más notables sobre el estudio de los beneficios de la confianza en la escuela es la realizada por Bryk y 
Schneider (2002). Ellos plantean que las escuelas son organizaciones compuestas por una amplia diversidad de individuos, entre los que se encuentra el director, los profesores, los estudiantes, los padres y apoderados. Las relaciones sociales que se desarrollan entre ellos se constituyen como recursos de mejoramiento y progreso para las escuelas, en especial para las que atienden a población socialmente vulnerable, en tanto enfrentan un escenario con mayores grados de complejidad.

Según Bryk y Schneider (2002), las investigaciones acerca de las escuelas efectivas advierten sobre el poder que tienen las relaciones sociales en el logro de los mejores resultados educacionales. Por ello, de acuerdo al autor, la confianza existente en las relaciones entre los profesionales de las escuelas (profesores y directores) y los padres de los alumnos, entre profesores y directores, se transforman en un recurso clave para instaurar y sostener los procesos de cambio a nivel de las organizaciones escolares. En este sentido, se puede postular que las relaciones sociales de confianza al interior de las escuelas pueden contribuir al proceso de implementación de las políticas educativas. Sobre todo si se considera que las organizaciones escolares, en la búsqueda del cumplimiento de sus propósitos, presentan un alto grado de dependencia entre los individuos que la componen, lo que al mismo tiempo les hace ser altamente vulnerables (Bryk y Schneider, 2002). En vista de ello, la confianza relacional adquiere una importancia especial en aquel contexto, convirtiéndose en un elemento crucial para avanzar en los procesos de mejoramiento de la escuela y de procesamiento y adopción de los cambios impulsados por las políticas educacionales. Bryk y Schneider (2002) sostiene que la confianza relacional tiene notables consecuencias para la organización escolar, ya que modera la sensación de incertidumbre y vulnerabilidad de los miembros de la organización; disminuye los costos de transacción ayudando en la resolución de problemas; contribuye al cumplimiento de roles y obligaciones de los miembros de la escuela; y a su vez, plantea un imperativo ético a los miembros de la organización para avanzar hacia el progreso de los estudiantes. 
Los autores construyen una perspectiva centrada en el mejoramiento de la educación en las escuelas desde la relación entre los actores de la comunidad educativa, superando otras visiones más centradas en el cambio estructural, o más enfocadas en la mejora de las prácticas docentes. Ellos plantean que las relaciones sociales y "la naturaleza del intercambio social, y los rasgos de la cultura local condicionan la capacidad de la escuela para mejorar" (Bryk y Schneider, 2002:5). La confianza forma parte de la cultura de la escuela, aportando a lubricar el funcionamiento diario de la escuela, funcionando como un recurso crítico para que los líderes generen un ambiente con planes de mejoramiento. Según las observaciones realizadas por los investigadores, esta confianza social aparece como especialmente importante en escuelas que atienden a niños socialmente desaventajados.

Existen distintas formas de confianza social: la confianza orgánica, la confianza contractual y la confianza relacional (Bryk y Schneider, 2002). La forma que toma la confianza dependería de la naturaleza de la institución social en la cual se inserta.

La confianza orgánica es la que opera cuando los individuos creen de manera incuestionable en una autoridad moral de una institución social. Suele darse en comunidades locales y en el caso de las escuelas religiosas fundamentalistas. En este tipo de organizaciones, la confianza es incondicional, se desarrolla en un contexto de completa obediencia. En cambio, la confianza contractual es más común en las instituciones modernas, se genera sobre la base que el intercambio social es material e instrumental. El contrato define las acciones básicas de las partes, por lo cual la invocación a la dimensión éticomoral, a diferencia de la confianza orgánica, no existe en este tipo de confianza. La confianza contractual supone acciones legales si alguien transgrede lo pactado en el contrato.

Por su parte, la confianza relacional se presenta en las escuelas y en los emprendimientos sociales (Bryk y Schneider, 2002). Tiene a su base la consideración de la relevancia de las relaciones sociales, por lo que el éxito de las operaciones organizacionales depende 
de los esfuerzos colectivos acerca del problema local detectado. En el contexto escolar, se genera un conjunto interrelacionado de dependencias mutuas entre todos los actores claves: estudiantes, profesores, directores, administradores y familiares. Esta dependencia genera un sentimiento de vulnerabilidad en los individuos, sobre todo para aquellos envueltos en relaciones asimétricas, como el caso de los profesores y familiares de estudiantes en situación de pobreza.

Cada uno de los actores de la organización escolar tiene obligaciones y expectativas acerca del cumplimiento de las obligaciones de otros, siendo necesaria la concordancia entre ambos para mantener altos niveles de confianza relacional. Este tipo de confianza, según los autores, estaría ubicado entre medio de la confianza orgánica y la confianza contractual.

Según los autores, el concepto de "confianza relacional es una teoría que tiene tres niveles” (Bryk y Schneider, 2002:I24). Uno intrapersonal, en el cual operan los criterios que permiten discernir acerca de las intenciones de los otros. Los principales criterios que operan son el respeto entre los individuos, la competencia en la ejecución de los roles formales de la responsabilidad de cada individuo, la estima personal para los demás y la integridad respecto a la consistencia entre lo que se dice y se hace. En el nivel interpersonal operan como factores estructurales la mutua dependencia y vulnerabilidad entre las partes de la organización escolar. Aquí se desarrolla un conjunto de roles entre director y profesor, entre profesores, entre padres y profesores y entre estudiantes. Estos dos niveles, el intrapersonal y el interpersonal, inciden en el organizacional, en el que existen fuerzas determinantes como el tamaño de la escuela, la estabilidad de la comunidad, la reputación institucional y factores que puede controlar la propia escuela, como el liderazgo y la asociación voluntaria. El elemento central de la confianza relacional en este nivel es la existencia de sincronía entre las obligaciones y expectativas a lo largo de la comunidad escolar.

Estos tres niveles tienen consecuencias claves en la organización escolar, haciendo sustentable la cultura de trabajo y facilitando su 
estructura. Esto a su vez incide en el ámbito instruccional, mejorando el aprendizaje de los estudiantes.

Según los autores, la confianza relacional no sólo tiene efectos en la escuela, sino que también tiene importantes consecuencias para las políticas educativas:

- En el desarrollo de nuevos profesores, ya que "para preparar mejores profesores, el ambiente del lugar de trabajo de las escuelas debe ser diferente. Debe existir un sentimiento compartido de la misión de educar a los niños” (Bryk y Schneider, 2002:136).

- En el desafío de construir confianza relacional en la comunidad escolar: para que la confianza relacional sea desarrollada y sostenible, adultos y estudiantes deben hacer sentido de su trabajo y comprender el propósito primario de la educación pública.

- En la centralidad del liderazgo del director como actor que fomenta la confianza relacional.

- En el apoyo de la relación de los profesores con los padres, en tanto se requiere de una comprensión empática de la situación de los padres por parte de los profesores, y se necesita que los profesores desarrollen habilidades para fortalecer a los adultos efectivamente.

- En la relación entre las reformas y las relaciones de confianza: la dependencia mutua no sólo existe al interior de la escuela, sino que también se expande hacia la red social de los actores internos y externos a la escuela. Los actores externos deben reconocer la vulnerabilidad sentida por los actores de la escuela y deben emprender acciones para reducirla, lo que ayuda a construir la confianza.

Uno de los mayores beneficios de la confianza es que favorece la efectividad de las escuelas. Existen estudios que asocian la confianza a la efectividad y productividad de la escuela: "que la confianza de una organización y su liderazgo tiene consecuencias en la productividad para las personas dentro de aquella organización es razonable y empíricamente apoyada en la literatura escolar" (Forsyth, Barnes y Adams, 2005:123). 
También es posible encontrar modelos que asocian la confianza con la efectividad de la escuela en materia de aprendizaje. En la Figura I se puede observar el modelo de escuela efectiva propuesto por Uline, Millar y Tschannen-Moran (1998).

Figura I: Modelo de escuela efectiva

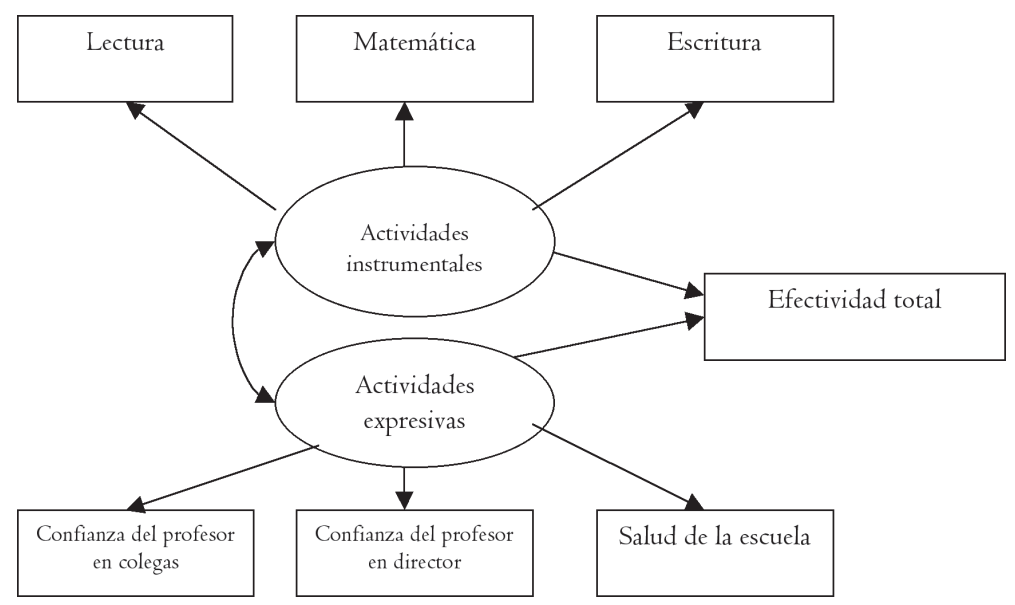

Fuente: Uline, Millar y Tschannen-Moran (1998:469)

El modelo propuesto por estos autores sigue a Etzioni (1975) y a Parsons (1960), combinando la adaptación y logro en sólo una función: las actividades instrumentales; y a su vez, combinando integración y latencia en una segunda función: las actividades expresivas. En el ámbito de las actividades expresivas, "la confianza es vista como un elemento vital en el buen funcionamiento de las organizaciones" (Uline, Millar y Tschannen-Moran, I998:470), como "el fundamento en el cual la efectividad de la escuela es construida" (Cunningham and Gresso, 1993:I2I). Según Uline, Millar y Tschannen-Moran (1998) la confianza es necesaria para la cooperación y comunicación efectiva, que son las bases para las relaciones productivas, y a la vez, construyen escuelas efectivas. Permite a las personas focalizarse en la tarea y por lo tanto, se trabaja y aprende con mayor efectividad. En cambio, la desconfianza causa que las personas se sientan incómodas y enfermen con facilidad. 
Existe evidencia empírica que relaciona la confianza de la escuela con su efectividad (Hoy, Tarter y Witkoskie, 1992). Los factores que permiten las relaciones de confianza también facilitan la efectividad de la escuela. Según Uline, Millar y Tschannen-Moran (1998), las relaciones interpersonales saludables, la apertura del clima de la escuela, una cultura escolar cohesiva, se encuentran relacionados a la confianza de la escuela y al logro de los estudiantes.

La autenticidad del comportamiento del director está relacionada a la confianza de la escuela en el director, así como también la autenticidad del comportamiento del profesor se relaciona con la confianza de la escuela en los colegas (Hoy y Henderson, 1983; Tschannen-Moran y Hoy, 1997).

También en el ámbito de las actividades expresivas se encuentra la salud de la escuela, "como un lugar donde las personas se sienten bien de estar y donde la escuela tiene la capacidad de cumplir su misión" (Uline, Millar y Tschannen-Moran, I998:470).

Dentro de las actividades instrumentales se puede destacar el logro del estudiante, que es la principal variable que refleja la efectividad de la escuela, pues aunque este es un constructo multidimensional, es imposible pensar en una escuela con alto desempeño si sus estudiantes no tienen un alto desempeño (Uline, Millar y Tschannen-Moran, I998). Aun así, podemos destacar que la efectividad de la escuela está relacionada a otros resultados como: "el crecimiento socio-emocional de los estudiantes, la satisfacción de los profesores, el uso eficiente de los recursos, la innovación, la adaptabilidad y el logro de las metas" (Cameron y Whetten, 1983, 1995; Hoy y Miskel, I99I, 1996).

En el estudio de Uline, Millar y Tschannen-Moran (1998) que fue aplicado a una muestra de 86 escuelas secundarias con un total de 2777 profesores, se llegó a la conclusión que tanto las actividades instrumentales como las expresivas ayudan a hacer escuelas efectivas. El logro de los estudiantes, la confianza de la escuela y la salud organizacional son todos indicadores de efectividad escolar (Uline, Millar y Tschannen -Moran, I998: 479). 
Por su parte, el estudio de Forsyth, Barnes y Adams (2005:123) plantea la importancia de la confianza de los padres y profesores para tres resultados escolares: eficacia colectiva de los profesores, estructura burocrática y desempeño académico. Si existe confianza de los profesores en sus colegas y en el director, basada en una historia de comportamiento consistente y con expectativas positivas, ellos creerán en su eficacia colectiva. La confianza tiene implicancias en la estructura burocrática, en tanto "puede reducir la necesidad de mecanismos burocráticos de control en organizaciones y controlar la proliferación de reglas y supervisión cercana" (Forsyth, Barnes y Adams 2005:127). Y por último, la confianza tiene efectos en el desempeño académico en tanto funciona como un lubricante para la cooperación, promoviendo la productividad. El estudio de estos investigadores se focalizó en 79 escuelas, utilizando las variables de Tschannen-Moran (1999) acerca de la confianza: benevolencia, fiabilidad, competencia, honestidad y apertura. El estudio se centró en el estudio de las percepciones de confianza, apoyando el razonamiento teórico de que el ambiente de confianza de la comunidad escolar es un poderoso predictor de las consecuencias de la escuela (Forsyth, Barnes y Adams, 2005).

Park, Henkin y Egley (2005) estudiaron la asociación entre el compromiso del equipo de profesores, el trabajo en equipo y la confianza. Ellos partieron de la idea que "el éxito de la reforma escolar depende, en parte, de la efectividad de los equipos de profesores"; "estos equipos descansan en la creatividad y habilidades de los profesores, quienes gustosamente contribuyen con su energía y lealtad a través de esfuerzos colectivos y procesos de equipo de trabajo" (Bolman y Deal, 1994; Duignan y Macpherson, I993; Glickman, 1993; Henkin, 2000; Pounder, 1998, citados en Park, Henkin y Egley, 2005:462). En el estudio de Park, Henkin y Egley (2005:466) se considera a la "confianza como un elemento esencial de la interacción social, siendo las transacciones de los equipos que están caracterizados por confianza interpersonal aquellos que promueven un ambiente en el cual los individuos se sienten libres de expresar sus ideas, resolver problemas y resolver diferencias de opinión”; 
"aquellas relaciones de trabajo caracterizadas por la confianza pueden fortalecer la cooperación, aumentar el compromiso organizacional y disminuir la tendencia al abandono". De esta forma, perciben que si los profesores no confían en el otro, no querrán colaborar. Los líderes de escuelas que funcionan con equipos preocupados de la dinámica de la confianza, toman los riesgos necesarios para desarrollar y fortalecer la confianza, lo que permite un trabajo en equipo con mayor efectividad (Park, Henkin y Egley, 2005:466). Los resultados del estudio, aplicado en 159 profesores, sugieren la importancia de la confianza como un elemento fundamental en la efectividad de los equipos de la escuela.

Por su parte, recordemos que las investigaciones de Bryk y Schneider (2002:106) revelan que "la confianza relacional de la comunidad escolar es un recurso clave para el mejoramiento", siendo un nivel base de confianza una condición para la capacidad que tiene la escuela de emprender nuevas iniciativas de reforma. Aunque también, según estos autores, existirían otros factores influyentes, tales como el contexto social y la composición del estudiantado. En el estudio, incluso después de controlar por diferencias entre escuelas en varios aspectos del contexto escolar, composición de los estudiantes y origen del profesor, se "encontró fuertes efectos vinculando cambios en la confianza relacional a los mejoramientos en la actividad productiva” (Bryke y Schneider, 2002:I I4). Según estos investigadores, la confianza relacional facilita el desarrollo de creencias, valores, rutinas organizacionales y comportamientos individuales que instrumentalmente afectan el compromiso de los estudiantes y el aprendizaje.

En Chile se han realizado estudios de efectividad escolar. El efectuado por Razcynski y Muñoz (2005) estudió las claves que hacen posible que una escuela vulnerable pueda obtener buenos resultados de aprendizaje, encontrando que uno de ellos es el que la escuela posea una cultura escolar positiva, con una fuerte ética del trabajo, un alto sentido identitario y un alto capital simbólico. De esta manera, los profesores de este tipo de escuela sentirían un alto compromiso e 
identificación con los objetivos de la escuela, que es contribuido, entre otros elementos, porque "el ambiente de la escuela es distendido, de confianza, de aceptación de ideas divergentes, de la evaluación y de la crítica" (Raczynski y Muñoz, 2005: 5). Otra de las claves que este estudio arroja es que la escuela cuente con un buen liderazgo institucional y pedagógico, el que sea legitimado y respetado por la comunidad escolar (Raczynski y Muñoz, 2005). En otras palabras, un director en el cual los profesores confien, quien a su vez confie en los profesores.

Pocas investigaciones se han realizado acerca de la confianza al interior de las escuelas en Chile. Particularmente, se puede mencionar la de Hurtado (2008), que a través de un análisis de regresión y factorial, caracteriza las relaciones de confianza de los profesores con el director y con sus mismos colegas, llegando al resultado de que "en las relaciones de confianza que se dan en la cultura escolar chilena predomina fuertemente lo afectivo, hasta el punto que incluso la evaluación del desempeño tiene que ver más con los afectos que con la competencia demostrada en el ejercicio del cargo" (Hurtado, 2008:12). Entre sus principales conclusiones advierte que las variables propias del soft management -como el clima, la confianza, la motivación, el liderazgo, el compromiso, entre otras- inciden directamente en el rendimiento académico, particularmente el clima, a través de la dimensión del énfasis académico, la que influye positiva y significativamente en el desempeño de los estudiantes. Otra de las conclusiones de este estudio es que se necesita estudiar las relaciones interpersonales dentro de las escuelas, "ya que a través de ellas es posible modificar el desempeño de los alumnos sin modificar las características inherentes al nivel socioeconómico o las características demográficas de los estudiantes” (Hurtado, 2008:37).

De esta forma, se puede apreciar que hay estudios que asocian la confianza a la efectividad de la escuela y a la capacidad de esta para enfrentar los cambios impulsados por las políticas educativas. Esta inmensa potencialidad de la confianza al interior de las escuelas merece ser estudiada y fortalecida a través de programas dirigidos a 
promover relaciones de confianza entre los miembros de la comunidad escolar.

\section{Conclusiones}

Las principales conclusiones de este artículo son:

- Las escuelas chilenas se encuentran actualmente enfrentando la exigencia de poner en marcha un sinnúmero de programas educativos, elaborados desde organismos centrales como respuesta a la demanda de la sociedad de mejoramiento de la calidad educativa. Esto por lo general se lleva a cabo sin que las escuelas cuenten con todas las capacidades para implementar de manera adecuada los programas para obtener los resultados que se esperan.

En este artículo hemos postulado que las capacidades de implementación de las políticas al interior de las escuelas, descansan fundamentalmente en la calidad de las relaciones sociales entre los actores que componen la comunidad escolar -especialmente entre profesores y director-en las que la presencia de la confianza actúa como lubricante de la relación e interacción cotidiana asociada a la labor como catalizador de trabajos y esfuerzos colectivos y como recurso motivador de esfuerzos por la mejora de la efectividad del colegio.

- El concepto de confianza ha sido poco trabajado por la teoría educativa, razón por la que resulta más fructífero adentrarse en la revisión de los aportes de la sociología en el estudio del tema. Sin embargo, hay que considerar que la mayor cantidad de literatura sobre la confianza se desprende de la teoría del capital social, existiendo en torno a esta teoría la mayor cantidad de estudios y acumulaciones disponibles.

Tomando en cuenta este escenario, la confianza puede ser comprendida desde distintos puntos de vista de acuerdo a la disciplina o tradición teórica desde la cual el investigador observe. No obstante, conviene 
tomar en cuenta que hay que superar visiones reduccionistas que conciban la confianza solamente en su dimensión psicológica o social. Por el contrario, es importante generar visiones lo más complejas posibles, considerando su dimensión interpersonal, organizacional y social.

En Chile, la confianza ha sido estudiada desde una perspectiva más general y no ha sido investigada lo suficiente en el ámbito educativo. Por ello, creemos que es importante profundizar en el estudio de los aspectos simbólicos de las relaciones interpersonales que contribuyen al funcionamiento de los colegios, teniendo en cuenta que las relaciones humanas en nuestro país se encuentran teñidas por la sensación de inseguridad y desconfianza hacia otro.

- La presencia de confianza en las relaciones entre profesores y director contribuye al buen funcionamiento de los colegios, sobre todo a aquellos que funcionan en un ambiente de alta exigencia o presión, con profesores y directores a menudo sobrecargados y con alta necesidad de trabajar de manera colectiva. Tal como hemos revisado en el análisis, sus beneficios incluyen la reducción de los costos de transacción, la promoción de los espacios de sociabilidad y trabajo en equipo, el incentivo de espacios de cooperación y trabajo colaborativo, los que inciden en la buena implementación de políticas y programas educativos, y en definitiva, en el buen funcionamiento de la organización escolar.

A lo largo de este análisis, se ha intentado aportar con algunas herramientas conceptuales para analizar las relaciones sociales al interior de la escuela, contribuyendo a la visibilización y comprensión de algunas pistas para descifrar la "caja negra" de la implementación de las políticas educacionales en las escuelas. Políticas que a menudo no obtienen los resultados esperados una vez que se implementan en las escuelas. 
La confianza interpersonal entre profesores y directores de escuelas: un pilar fundamental para la implementación de políticas y programas educativos - Alicia Razeto P.

\section{Referencias bibliográficas}

Baron, S.; Field, J. y Schuller, T. (2000) (editores). Social Capital. Critical Perspectivas. Oxford University Press, Nueva York.

Brewster, C. y Railsback, J. (2003). Building trusting relationships for school improvement: implications for principals and teacher. Northwest regional educational laboratory.

Bryk y Schneider (2002). Trust in schools: a core resource for improvement. Russell Sage Foundation, New York.

Forsyth, P.; Barnes, L. y Adams, C. (2006). Trust-effectiveness patterns in schools en Journal of Educational Administration Vol. $44 \mathrm{~N}^{\circ} 2$ (pp. I22-I4I).

Fukuyama, F. (1996). Confianza (trust). Las virtudes sociales y la capacidad de generar prosperidad. Editorial Atlántida, Buenos Aires.

Hooghe, M. y Stolle, D. (2003). Generating Social Capital. Civil society and institutions in comparative perspective. Palgrave Macmillan, New York.

Hurtado, José (2008). Gestión de instituciones escolares: la cultura escolar cbilena y la influencia de las variables del soft management en el rendimiento académico. Tesis para optar al grado de Magíster en Ciencias de la Ingeniería. Profesor supervisor: Nicolás Majluf. Pontificia Universidad Católica de Chile.

Kramer, R. (1999). Trust and distrust in organizations: emerging perspectives, enduring questions.

Luhmann, N. (1979). Power and trust. Chichester: Wiley.

Luhmann, N. (1996). Confianza. Anthropos, Barcelona.

Misztal, B. (1996). Trust in modern societies: the search for the bases of social order. Blackwell. Cambridge, U.K.

Moye, M.; Henkin, A. y Egley, R. (2005). "Teacher-principal relationships. Exploring linkages between empowerment and interpersonal trust", en Journal of Educational Administration. Vol 43, No 3 (pp.: 260 - 277).

Park, S. y Henkin, A. (2005). "Teacher team commitment, teamwork and trust: exploring associations" en Journal of Educational Administration. Vol. $43 \mathrm{~N}^{\circ}$ 5 (pp. 462 - 479).

Programa de Naciones Unidas para el Desarrollo (1998). Las Paradojas de la Modernización, Informe sobre Desarrollo Humano en Chile. Santiago de Chile.

Raczynski, D.; Serrano, C. (2005). "Programas de superación de la pobreza y capital social. Evidencias y aprendizajes de la experiencia en Chile" en Libro de la CEPAL Aprender de la experiencia. 
Disponible en: http://www.asesoriasparaeldesarrollo.cl/?q=areastrabajo/ ciudadania_capital, visitado el I8 de julio de 2008.

Raczynski, D.; Muñoz, G. (2005). Escuelas efectivas en sectores en pobreza. ¿Quién dijo que no se puede?

Seligman, A. (1997). The problem of trust. Princeton University Press, Princeton, NJ.

Sztompka, P. (1999). Trust: a sociological theory. Cambridge University Press, Cambridge.

Uline, C., Miller, D. y Tschannen-Moran, M. (1998). School Effectiveness: The Underlying Dimensions en Educational Administration Quarterly Vol 34, $\mathrm{N}^{\circ}$ (October 1998) (pp. 462-483).

Uslaner, E. (2002). The moral foundations of trust. Cambridge University Press, Cambridge. 